\title{
INVESTIGATION OF CHANGES AND DEVELOPMENT OF MATHEMATICAL MODEL FOR A DRILLING VESSEL
}

\author{
Kramar Vadim \\ Sevastopol State University, 33, University St., Sevastopol, 299053, Russia
}

\begin{abstract}
The article discusses the technique of construction of the generalized mathematical model of spatial movement of the drilling vessel, taking into account the mutual influence of different types of movement.

Ships of various design and deadweight are used in today's worldwide practice for marine drilling aimed at probing or extracting minerals. There are automatic keeping systems over the drilling site in some of them. However, the information contained in express materials and advertisement messages disables from figuring out on what principles these systems are built, and, moreover, what the applied technical facilities comprise and what features it has. These concerns require consideration in respect of theory as well as applying to technical implementation.

We have an aggregated representation of drilling vessel spacial movement, figuring on mutual influence of various types of motion (rolling, pitching, heaving motions, vessel yaw and horizontal plane transfers), as well as dimensional changes of the vessel depending on the kind of performed activity.
\end{abstract}

Keywords: drilling vessel;mathematical model;automatic keeping systems
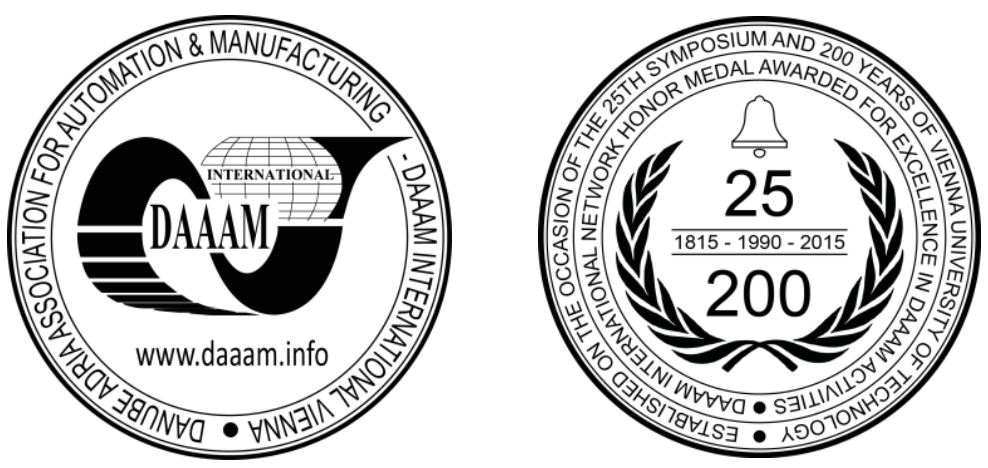

This Publication has to be referred as: Kramar, V[adim] (2016). Investigation of Changes and Development of Mathematical Model for a Drilling Vessel, Proceedings of the 26th DAAAM International Symposium, pp.0049-0055, B. Katalinic (Ed.), Published by DAAAM International, ISBN 978-3-902734-07-5, ISSN 1726-9679, Vienna, Austria DOI:10.2507/26th.daaam.proceedings.007 


\section{Introduction}

Ships of various design and deadweight are used in today's worldwide practice for marine drilling aimed at probing or extracting minerals. There are automatic keeping systems over the drilling site in some of them. The principles of these systems and the applied technical facilities comprise and features require consideration in respect of theory as well as applying to technical implementation. Information about the mathematical description of the drilling vessel as a control object of the automatic deduction contained in [1-5] do not contain analytical relation ships hydroaerodynamics characteristics and do not reveal the principles of such systems.

The following issues are crucial to automatic keeping system design: the development the mathematical models [6,7] of drilling vessel, that would figure on the peculiarities of vessel constructions, drilling equipment and technological operations in the course of drilling activity; ratio deduction for calculating outer forces and things affecting a drilling vessel by its dislocation regarding the wind, the stream, sea swells; the investigation of space movement changes under keeping conditions; the selection of active steering means and ratio deduction for determining thrust forces of active steering means, intended to keep a vessel in a settled location at every instant; the development of guidelines for building a measuring system on determining horizon transfers in the standard of rest, static or aligned with a vessel ; the choice of adjustable profiles and the assessment of the required measurement accuracy; the identification of the automatic keeping system structure. It is the aboved-mentioned questions that are enquired into in the actual research.

The movement of a drilling vessel by keeping it on a drilling point is conditioned not only by a sort or a nature of outer impact (of the wind, the stream, sea disturbance, thrust powers of active steering means), but also by a kind of activity, whereas the latter can alter dynamic features of the whole vessel. Owing to this the whole time, during which the vessel should be kept on a settled point, may be divided into four periods 1) the initial stage; 2) the preliminary stage; 3 ) the drilling stage ; 4) the ultimate stage.

In the process of keeping on the drilling site a drilling vessel may have five dynamic models, different from each other. All the distinctive peculiarities of a drilling vessel by keeping it on the drilling site come out at the preliminary stage and at the drilling one. Therefore these two models are of the greatest interest. They allow to determine what effect the performed drilling activity makes on vessel movement changes under keeping conditions.

Ratios of force and instant projection on reference axes aligned with the vessel are included in this research. We have an extended representation of drilling vessel special movement changes, figuring on the above-mentioned peculiarities of the periods during which drilling operations are carried out.

\section{Mathematical model of a for drilling vessel}

Let us introduce for consideration the following systems of coordinate, which characterize spacial position of the vessel and which is analog as [8].

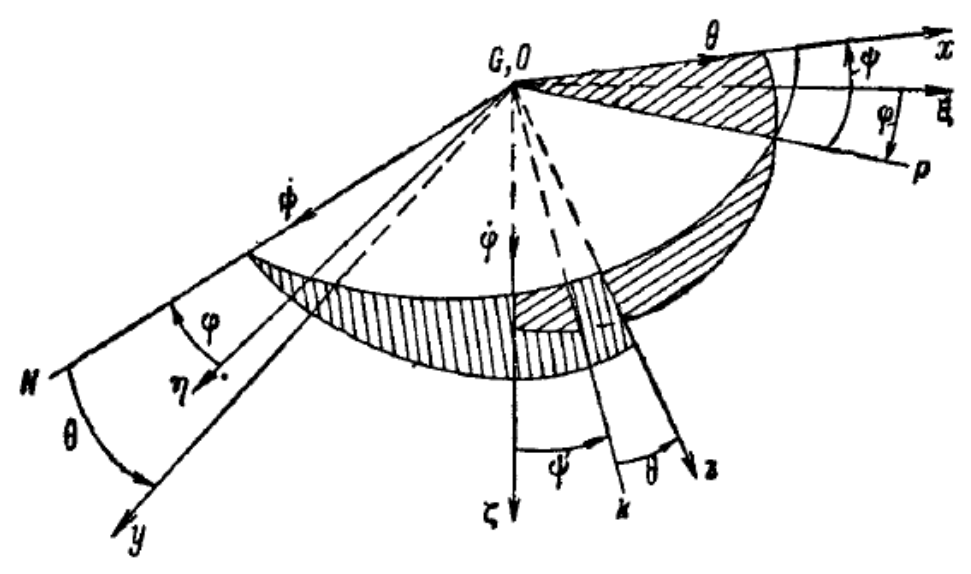

Fig. 1. Coordinate systems

The movement of the vessel may be represented as consisting of translational motion with the starting point $\mathrm{G}$ of the coordinate system, closely related to the vessel, and rotation around the point G. Vessel movement equations comprise movement equations of the point $\mathrm{G}$ dynamics, which coincides with the vessel gravity center at the moment, equations of rotation movement towards the point $\mathrm{G}$ and equations of the gravity center dislocation in the course of drilling operations.

Let us examine the movement of the vessel on the free infinite liquid surface. Let us disregard liquid elasticity(ideal liquid).To obtain the equation of solid body movement in the ideal infinite liquid, we shall examine the movement of the system "body+liquid in the vacuum" as it is carried out in [I]. Equations of movement of the examined system may be formulated as: 


$$
\frac{d \vec{R}}{d t}=\frac{d(\vec{Q}+\vec{B})}{d t}=\vec{F}+\vec{F}^{*}, \quad \frac{d \vec{N}}{d t}=\frac{d(\vec{K}+\vec{I})}{d t}=\vec{M}+\vec{M}^{*},
$$

where $\vec{Q}$ and $\vec{B}$ are the main motion quantity vectors in conformity with the vessel and liquid; $\vec{K}$ and $\vec{I}$ are the main motion quantity instants in conformity with the vessel and liquid in relation to the reference point; $\vec{F}$ is the main outer force vector; $\vec{M}$ is the main outer force instant; $\vec{F}^{*}$ is the motion quantity vector of the particles, thrown off the body surface within a unit of time; $\vec{M}^{*}$ is the motion quantity instant vector of the particles, thrown off within a unit of time. The vectors $\vec{F}^{*}$ and $\vec{M}^{*}$ can present as:

$$
\vec{M}^{*}=\sum_{v=1}^{n} \vec{r}_{v} \times \frac{d m_{v}}{d t} \vec{u}_{v}, \quad \vec{F}^{*}=\sum_{v=1}^{n} \frac{d m_{v}}{d t} \vec{u}_{v},
$$

where $\vec{u}_{v}$ is the absolute rate of the particles thrown off by a point having the mass $m_{v}, \vec{r}_{v}$ is the position vector of the point $m_{v}$ with respect to the static coordinate system, the plane of whichcoincides with theundisturbed freesurface of the liquid, and one axisis directed downwardalong theborehole (well).

For the velocity $\vec{u}_{v}$ we can wright the formula $\vec{u}_{v}=\vec{v}+\vec{\omega}+\vec{r}_{v}$, and for the projections on the axes of the static coordinate system the following formulae may be brought in:

$$
\begin{aligned}
& u_{v x}=v_{g x}+\omega_{y} z_{v}=v_{g x}+\dot{\psi} z_{v}, \\
& u_{v y}=v_{g y}-\omega_{x} z_{v}=v_{g y}-\dot{\theta}_{v}, \\
& u_{v z}=v_{g z} .
\end{aligned}
$$

Now, resulting from the expression(2), we obtain formulae for projections of motion quantity and motion quantity instants of the particles, thrown off the body surface within a unit of time $F_{x}^{*}, F_{y}^{*}, F_{z}^{*}, M_{x}^{*}, M_{y}^{*}, M_{z}^{*}$ : and proceding to the system of coordinates closely related to the body, instead of(1) we obtain:

$$
\frac{d \vec{R}}{d t}+\vec{\omega} \times \vec{R}=\vec{F}+\vec{F}^{*}, \quad \frac{d \vec{N}}{d t}+\vec{\omega} \times \vec{N}+\vec{v} \times \vec{R}=\vec{M}+\vec{M}^{*}
$$

where $\vec{v}$ is the vector of reference point rate of the static system; $\vec{\omega}$ is the vector of body rotation angular velocity.Writing the expression (4) in the projections on the coordinate axes of the coordinate system associated with the ship where the plane coincides with the plane of equilibrium waterline of the vessel, and the axis passing through its center of gravity, we obtain the general equation of motion of a rigid body

$$
\begin{aligned}
& d R_{x} / d t+\omega_{y} R_{z}-\omega_{z} R_{y}=F_{x}+F_{x}^{*}, \\
& d R_{y} / d t+\omega_{z} R_{x}-\omega_{x} R_{z}=F_{y}+F_{y}^{*}, \\
& d R_{z} / d t+\omega_{x} R_{y}-\omega_{y} R_{x}=F_{z}+F_{z}^{*}, \\
& d N_{x} / d t+\omega_{y} N_{z}-\omega_{z} N_{y}+v_{y} R_{z}-v_{z} R_{y}=M_{x}+M_{x}^{*}, \\
& d N_{y} / d t+\omega_{z} N_{x}-\omega_{x} N_{z}+v_{z} R_{x}-v_{x} R_{z}=M_{y}+M_{y}^{*}, \\
& d N_{z} / d t+\omega_{x} N_{y}-\omega_{y} N_{x}+v_{x} R_{y}-v_{y} R_{x}=M_{z}+M_{z}^{*} .
\end{aligned}
$$

To determine the integrate vectors $\vec{R}$ and $\vec{N}$ in (5) let us use the formulae known from theoretical mechanics:

$$
\begin{aligned}
& \partial T_{c} / \partial v_{x}=R_{x}, \partial T_{c} / \partial v_{y}=R_{y}, \partial T_{c} / \partial v_{z}=R_{z}, \\
& \partial T_{c} / \partial \omega_{x}=N_{x}, \partial T_{c} / \partial \omega_{y}=N_{y}, \partial T_{c} / \partial \omega_{z}=N_{z},
\end{aligned}
$$

where $T_{c}=T+T_{1}$ is motional energy of the system "body+liquid", $v_{x}, v_{y}, v_{z}$ are integrate velocities of translational motion in the standard of rest related to the vessel $\omega_{x}, \omega_{y}, \omega_{z}$ are integrate velocities of rotational motion in the same standard of rest. 
The amount of liquid motional energy is expressed by the formula $T_{1}=\frac{\rho}{2} \iiint_{V} v^{2} d V$, where the integral is taken along the liquid volume $V$. Let us draw optional static surface $\Sigma$ in the space surrounding the surface $\Omega$ of the moving vessel. Let us examine the integral of the volume $V$, encompassed by the surfaces $\Sigma$ and $\Omega$. According to Green theorem

$$
\iiint_{V} v^{2} d V=-\iint_{\Omega} \Phi \frac{\partial \Phi}{\partial n} d \Omega+\iint_{\Sigma} \varphi \frac{\partial \varphi}{\partial n} d \Sigma
$$

where $\varphi$ is potential of unstable motion liquid velocities, $\Phi$ is potential of disturbed liquid motion velocities. It issues from the vessel motion.

On removing the surface $\Sigma$ to perpetuity the last integral gravitates towards the zero, and for motional energy of all unlimited extent of liquid beyond the surface $\Omega$, we obtain

$$
T_{1}=\frac{\rho}{2} \iiint_{V} v^{2} d V=-\frac{\rho}{2} \iint_{\Omega} \Phi \frac{\partial \Phi}{\partial n} d \Omega
$$

It is apparent from (7) that it is necessary to figure out the liquid velocity potential $\Phi$ to determine motional energy of the liquid. In the static coordinate system liquid velocity potential is the solution to Laplace equation

$$
\Delta \varphi=\partial^{2} \varphi / \partial \xi^{2}+\partial^{2} \varphi / \partial \eta^{2}+\partial^{2} \varphi / \partial \zeta^{2}=0
$$

and it complies with the condition of attached liquid flow over the hull on the surface $\Omega$

$$
\frac{\partial \varphi}{\partial n}=\vec{v} \vec{n}
$$

where $\vec{v}$ is vessel hull certain point rate, $\vec{n}$ is normal line to the vessel hull in this point.

On the free liquid surface the potential $\varphi$ satisfies the terminal condition:

$$
-\frac{\partial \varphi}{\partial \varsigma}+\frac{1}{g} \frac{\partial^{2} \varphi}{\partial t^{2}}=0 \text { with } \varsigma=0
$$

On account of linearity of the Laplasian equation and terminal conditions, in the case of unstable liquid motion it is possible to make the velocity potential look like:

$\varphi(\xi, \eta, \varsigma, t)=\Phi_{1}(\xi, \eta, \varsigma, t)+\Phi_{b}(\xi, \eta, \varsigma, t)$

where $\Phi_{b}$ is the potential of spontaneous wave system rates on the liquid surface, $\Phi_{1}$ is the potential of disturbed liquid motion velocity, resulting from vessel motions and spontaneous wave system.

Velocity distribution of the vessel as a solid body may be determined setting a body point rate and angular velocity of its rotation. On denoting as $\vec{v}_{g}$ the rate vector of the reference point $G$ of the standard of rest related to the vessel and as $\vec{\omega}$ the angular velocity vector of vessel rotation, we obtain for arbitrary vessel point rate

$$
\vec{v}=\vec{v}_{g}+\vec{\omega} \times \vec{r}
$$

where $\vec{r}$ is the radius-vector of this arbitrary point with respect to the point $G$. We obtain in the projections on the reference axes

$$
\begin{aligned}
& v_{x}=v_{g x}+\omega_{y} z-\omega_{z} y, \\
& v_{y}=v_{g y}+\omega_{z} x-\omega_{x} z, \\
& v_{z}=v_{g z}+\omega_{x} y-\omega_{y} x .
\end{aligned}
$$


Let us denote the cosines of the angles formed by the normal line to the surface of the vessel hull with reference axes

$\cos \left(n^{\wedge}, x\right)=\alpha, \cos \left(n^{\wedge}, y\right)=\beta, \cos \left(n^{\wedge}, z\right)=\gamma$

In that case for normal components of a point rate on the surface of the vessel hull we obtain

$v_{n}=v_{x} \alpha+v_{y} \beta+v_{z} \gamma$

Flow condition on the body surface for the potential $\Phi_{1}$ will now be written as the following

$\frac{\partial \Phi_{1}}{\partial n}=v_{g x} \alpha+v_{g y} \beta+v_{g z} \gamma+\omega_{x}(y \gamma-z \beta)+\omega_{y}(z \alpha-x \gamma)+\omega_{z}(x \beta-y \alpha)-\frac{\partial \Phi_{b}}{\partial n}$

where it is apparent, that the function $\Phi_{1}$ may be presented as

$\Phi_{1}=v_{g x} \varphi_{1}+v_{g y} \varphi_{2}+v_{g z} \varphi_{3}+\omega_{x} \varphi_{4}+\omega_{y} \varphi_{5}+\omega_{z} \varphi_{6}+\Phi_{0}=\Phi+\Phi_{0}$

where $\Phi$ is the potential of fended progressive waves velocities.

All the functions $\varphi_{i}$ should satisfy the Laplasian equation and derivative boundedness condition on the perpetuity. On the vessel hull surface they should satisfy the conditions:

$$
\frac{\partial \varphi_{1}}{\partial n}=\alpha, \frac{\partial \varphi_{2}}{\partial n}=\beta, \frac{\partial \varphi_{3}}{\partial n}=\gamma, \frac{\partial \varphi_{4}}{\partial n}=\gamma_{y}-z \beta, \frac{\partial \varphi_{5}}{\partial n}=z \alpha-x \gamma, \frac{\partial \varphi_{6}}{\partial n}=x \beta-y \alpha, \frac{\partial \Phi_{0}}{\partial n}=-\frac{\partial \Phi_{b}}{\partial n}
$$

The conditions (11) are independent of the vectors $\vec{v}_{g}$ and $\vec{\omega}$, so all the six functions $\varphi_{i}$ are determined by the hull surface shape and the choice of a coordinate system related to the vessel. In view of terminal condition on the liquid surface the functions $\varphi_{i}(x, y)$ depend on oscillation frequency and rate of sailing.

In problems about balancing on the water surface the following congruence may occur

$$
\lambda_{j k}-\frac{i}{\sigma_{k}} \mu_{j k}=-\rho \iint_{\Omega} \varphi_{j} \frac{\partial \varphi_{k}}{\partial n} d \Omega \quad(j, k=1,2, \ldots, 6)
$$

where $\sigma_{k}$ is pitching frequency; $\lambda_{j k}$ and $\mu_{j k}$ are hydrodynamic coefficients which determine inertia and damping forces of wave nature.

On denoting $v_{g x}=v_{1}, v_{g y}=v_{2}, v_{g z}=v_{3}, \omega_{x}=v_{4}, \omega_{y}=v_{5}, \omega_{z}=v_{6}$, From (11) we obtain

$$
\Phi=\sum_{i=1}^{6} v_{i} \varphi_{i}
$$

For liquid motional energy regarding (13) and (14) we obtain

$$
T_{1}=\frac{1}{2} \sum_{j=1}^{6} \sum_{k=1}^{6}\left(\lambda_{j k}-\frac{i}{\sigma_{k}} \mu_{j k}\right) v_{j} v_{k}
$$

The kinetic energy of the solid mass, symmetrically about the longitudinal vertical plane $\left(\lambda_{j k}=\lambda_{k j}, \mu_{j k}=\mu_{k j}\right)$, is given by

$$
\begin{aligned}
T= & \frac{1}{2}\left[m\left(v_{1}^{2}+v_{2}^{2}+v_{3}^{2}\right)+2 m v_{1}\left(v_{5} z_{c}-v_{6} y_{c}\right)+2 m v_{2}\left(v_{6} x_{c}-v_{4} z_{c}\right)+\right. \\
& \left.+2 m v_{3}\left(v_{4} y_{c}-v_{5} x_{c}\right)+J_{x} v_{4}^{2}+J_{y} v_{5}^{2}+J_{z} v_{6}^{2}\right]
\end{aligned}
$$

where $J_{x}, J_{y}, J_{z}$ are axial moments of body inertia $, x_{c}, y_{c}, z_{c}$ are the points of body inertia center. Finally, we obtain the kinetic energy of the solid+ liquid 


$$
\begin{aligned}
T_{c}= & \frac{1}{2}\left[m+\lambda_{11}-\frac{i}{\sigma_{k}} \mu_{11}\right) v_{g x}^{2}+\left(m+\lambda_{22}-\frac{i}{\sigma_{k}} \mu_{22}\right) v_{g y}^{2}+\left(m+\lambda_{33}-\frac{i}{\sigma_{k}} \mu_{33}\right) v_{g z}^{2}+ \\
& \left.+\left(J_{x}+\lambda_{44}-\frac{i}{\sigma_{k}} \mu_{44}\right) \omega_{x}^{2}+\left(J_{y}+\lambda_{55}-\frac{i}{\sigma_{k}} \mu_{55}\right) \omega_{z}^{2}\right]+m v_{g x}\left(\omega_{y} z_{c}-\omega_{z} y_{c}\right)+ \\
& +m v_{g y}\left(\omega_{z} x_{c}-\omega_{x} z_{c}\right)+m v_{g z}\left(\omega_{x} y_{c}-\omega_{y} x_{c}\right)+\lambda_{26} v_{g y} \omega_{z}+\lambda_{35} v_{g z} \omega_{y} .
\end{aligned}
$$

Plugging the obtained formula for motional energy into the formulae (6), we find outthe expression for the components of vectors $R_{x}, R_{y}, R_{z}, N_{x}, N_{y}, N_{z}$.

Here and further the mark $g$ in the projections of translational motion velocity $v$ is omitted to simplify the formula.

To go further we shall discuss a series of assumptions. Let us assume that: 1) The angles $\theta$ and $\psi$ are small, therefore $\sin \theta=\theta, \quad \sin \psi=\psi, \cos \theta=\cos \psi=1 ; 2)$ movement through the points $\psi$ and $\theta$ are forced and cyclic with oscillation frequency $\sigma_{k}$, depending on the regular waves frequency $\sigma$ and the vessel velocity $v: \sigma_{k}=\sigma-v k \cos \chi$, where $k$ is the module of sea wave shape, $\chi$ - the angle between the direction of the vessel and that of wave advance; 3) movement along the vertical and transverse horizontal coordinates in the fixed coordinate system consists of the cyclic component ( frequency $\sigma_{k}$ ) and the noncyclic one; 4) we disregard damping fluid properties towards the axis $O \xi ; 5)$ the elements containing products of the values $\psi, \dot{\psi}, \theta, \dot{\theta}, \dot{\varphi}, \ddot{\varphi}$ are small and can be disregarded. In accordance with the assumptions accepted we can formulate:

$\psi=\psi_{0} e^{i \sigma_{k} t}, \theta=\theta_{0} e^{i \sigma_{k} t}, \varsigma_{g}=\varsigma_{c}+\varsigma_{b}=\varsigma_{c}+\varsigma_{0} e^{i \sigma_{k} t}, \eta_{g}=\eta_{c}+\eta_{b}=\eta_{c}+e^{i \sigma_{k} t}$

where $\psi_{0}, \theta_{0}, \varsigma_{0}, \eta_{0}$ are complex amplitudes, $\varsigma_{c}, \eta_{c}$ are noncyclic components of the movement. For point change rates and angles we obtain the formula

$\dot{\psi}=i \sigma_{k} \psi, \dot{\theta}=i \sigma_{k} \theta, \dot{\varsigma}_{g}=\dot{\zeta}_{c}+i \sigma_{k} \varsigma_{b}, \dot{\eta}_{g}=\dot{\eta}_{c}+i \sigma_{k} \eta_{b}$

Having small values $\theta, \psi, \dot{\varphi}$ for angular and linear rates in the related standard of rest we obtain

$$
\begin{aligned}
\omega_{x}= & \dot{\theta} ; \omega_{y}=\dot{\psi} ; \omega_{z}=\dot{\varphi} ; \\
v_{x}= & a_{1} \dot{\xi}_{g}+a_{2} \dot{\eta}_{g}+a_{3} \dot{\zeta}_{g}=\dot{\xi}_{g} \cos \varphi+\dot{\eta}_{c} \sin \varphi+i \sigma_{k}\left(\eta_{b} \sin \varphi+\sigma_{k}^{-2} \dot{\psi} \dot{\zeta}_{g}\right) ; \\
v_{y}= & b_{1} \dot{\xi}_{g}+b_{2} \dot{\eta}_{g}+b_{3} \dot{\zeta}_{g}=-\dot{\xi}_{g} \sin \varphi+\dot{\eta}_{c} \cos \varphi+i \sigma_{k}\left(\eta_{b} \cos \varphi+\varsigma_{b} \theta\right)+\theta \dot{\zeta}_{c} ; \\
v_{z}= & c_{1} \dot{\xi}_{g}+c_{2} \dot{\eta}_{g}+c_{3} \dot{\zeta}_{g}=(\psi \cos \varphi+\theta \sin \varphi) \dot{\xi}_{g}+(\psi \sin \varphi-\theta \cos \varphi) \dot{\eta}_{g}+\dot{\zeta}_{c}+ \\
& +i \sigma_{k}\left[\eta_{b}(\psi \sin \varphi-\theta \cos \varphi)+\varsigma_{b}\right] .
\end{aligned}
$$

Writing at small values $\theta, \psi, \dot{\varphi}$ the expressions for the angular and linear velocity related coordinate system, substituting them in to the expression for $R_{x}, R_{y}, R_{z}, N_{x}, N_{y}, N_{z}$, and taking into account only thereal partsget

$$
\begin{aligned}
& R_{x}=\left(m+\lambda_{11}\right) v_{x}+m z_{c} \dot{\psi} ; \\
& R_{y}=\left(m+\lambda_{22}\right) v_{y}-\left(m z_{c}-\lambda_{24}\right) \dot{\theta}+\left(\mu_{22} \zeta_{b}+\mu_{24}\right) \theta+\lambda_{26} \dot{\varphi}+\mu_{22} \eta_{b} \cos \varphi \\
& R_{z}=\left(m+\lambda_{33}\right) v_{z}+\lambda_{35} \dot{\psi}+\left(\mu_{33} \eta_{b} \sin \varphi+\mu_{35}\right) \psi-\mu_{33} \eta_{b} \theta \cos \varphi+\mu_{33} \zeta_{b} \\
& N_{x}=\left(J_{x}+\lambda_{44}\right) \dot{\theta}-\left(m z_{c}-\lambda_{24}\right) v_{y}+\mu_{24}\left(\eta_{b} \cos \varphi+\theta \zeta_{b}\right)+\mu_{44} \theta \\
& N_{y}=\left(J_{y}+\lambda_{55}\right) \dot{\psi}+\left(\mu_{55}+\mu_{35} \eta_{b} \sin \varphi\right) \psi+m z_{c} v_{x}+\lambda_{35} v_{z}-\mu_{35} \eta_{b} \theta \cos \varphi+\mu_{35} \xi_{b} \\
& N_{z}=\left(J_{z}+\lambda_{66}\right) \dot{\varphi}+\lambda_{26} v_{y}+\mu_{26}\left(\eta_{b} \cos \varphi+\zeta_{b} \theta\right)
\end{aligned}
$$

Substituting (21) inequation (5), provided that the weight of the vessel, its hydrodynamic characteristics and moments of inertia change over time and considering the relationship for $F_{x}^{*}, F_{y}^{*}, F_{z}^{*}, M_{x}^{*}, M_{y}^{*}, M_{z}^{*}$, we obtain a generalized model ofthe interrelatedmovementsof the drillingvesselon a regularwaves thatfor the studyof hisspeakersneed to be supplementedby equationsfor forcedcomponentsof vertical andhorizontaltransverse motion ofthe shipon a regular waves 


$$
\begin{gathered}
\left(m+\lambda_{11}\right) \dot{v}_{x}+\dot{\lambda}_{11} v_{x}+m z_{c} \ddot{\psi}+\left[m \dot{z}_{c}+\left(m+\lambda_{33}\right) v_{z}+\mu_{33} \varsigma_{b}\right] \dot{\psi}- \\
-\left[\left(m+\lambda_{22}\right) v_{y}+\mu_{22} \eta_{b} \cos \varphi\right] \dot{\varphi}=F_{x}, \\
\left(m+\lambda_{22}\right) \dot{v}_{y}+\dot{\lambda}_{22} v_{y}-\left(m z_{c}-\lambda_{24}\right) \ddot{\theta}+\left[\mu_{22} \varsigma_{b}+\mu_{24}-m \dot{z}_{c}+\dot{\lambda}_{24}-\left(m+\lambda_{33}\right) v_{z}-\mu_{33} \varsigma_{b}\right] \dot{\theta}+ \\
+\left(\dot{\mu}_{22} \varsigma_{b}+\mu_{22} \dot{\varsigma}_{b}+\dot{\mu}_{24}\right) \theta+\lambda_{26} \ddot{\varphi}+\left[\dot{\lambda}_{26}-\mu_{22} \eta_{b} \sin \varphi+\left(m+\lambda_{11}\right) v_{x}\right] \dot{\varphi}+\left(\dot{\mu}_{22} \eta_{b}+\mu_{22} \dot{\eta}_{b}\right) \cos \varphi=F_{y},
\end{gathered}
$$

$\left(m+\lambda_{33}\right) \dot{v}_{z}+\dot{\lambda}_{33} v_{z}+\lambda_{35} \ddot{\psi}+\left[\dot{\lambda}_{35}+\mu_{33} \eta_{b} \sin \varphi+\mu_{35}-\left(m+\lambda_{11}\right) v_{x}\right] \dot{\psi}+$

$+\left(\dot{\mu}_{33} \eta_{b} \sin \varphi+\mu_{33} \dot{\eta}_{b} \sin \varphi+\dot{\mu}_{35}\right) \psi+\left[\left(m+\lambda_{22}\right) v_{y}+\left(\mu_{22}-\mu_{33}\right) \eta_{b} \cos \varphi\right] \dot{\theta}-$

$-\left(\dot{\mu}_{33} \eta_{b} \cos \varphi+\mu_{33} \dot{\eta}_{b} \cos \varphi\right) \theta+\mu_{35} \dot{\varsigma}_{b}+\dot{\mu}_{35} \varsigma_{b}=F_{z}$,

$$
\left(J_{x}+\lambda_{44}\right) \ddot{\theta}+\left[J_{x}+\dot{\lambda}_{44}+\mu_{24} \varsigma_{b}+\mu_{44}+\left(m z_{c}-\lambda_{24}\right) v_{z}-\dot{m} z_{c}^{2}\right] \dot{\theta}+
$$$$
+\left[\dot{\mu}_{24} \varsigma_{b}+\mu_{24} \dot{\zeta}_{b}+\dot{\mu}_{44}-\mu_{33} \eta_{b} \cos \varphi v_{y}-\left(\mu_{24}+\mu_{22} \varsigma_{b}\right) v_{z}\right] \theta-\left(m z_{c}-\lambda_{24}\right) \dot{v}_{y}-
$$$$
-\left[m \dot{z}_{c}-\lambda_{24}-\left(\mu_{33} \eta_{b} \sin \varphi+\mu_{35}\right) \psi-\left(\lambda_{33}-\lambda_{22}\right) v_{z}-\left(\lambda_{35}+\lambda_{26}\right) \dot{\psi}-\mu_{33} \varsigma_{b}\right] v_{y}-
$$

$-\left[\mu_{24} \eta_{b} \sin \varphi+\left(\lambda_{35}+\lambda_{26}\right) v_{z}+\mu_{35} \varsigma_{b}+m z_{c} v_{x}\right] \dot{\varphi}+\left(\mu_{24} \dot{\eta}_{b}+\dot{\mu}_{24} \eta_{b}-\mu_{22} \eta_{b} v_{z}\right) \cos \varphi=M_{x}$,

$\left(J_{y}+\lambda_{55}\right) \ddot{\psi}+\left(\dot{J}_{y}+\dot{\lambda}_{55}+\mu_{55}+\mu_{35} \eta_{b} \sin \varphi+m z_{c} v_{z}-\lambda_{35} v_{x}-\dot{m} z_{c}^{2}\right) \dot{\psi}+$

$+\left(\dot{\mu}_{55}+\dot{\mu}_{35} \eta_{b} \sin \varphi+\mu_{35} \dot{\eta}_{b} \sin \varphi-\mu_{33} \eta_{b} \sin \varphi v_{x}-\mu_{35} v_{x}\right) \psi+m z_{c} \ddot{v}_{x}+\left[m \dot{z}_{c}+\right.$

$\left.+\left(\lambda_{11}-\lambda_{33}\right) v_{z}+\mu_{33}\left(\eta_{b} \cos \varphi \cdot \theta-\varsigma_{b}\right)\right] v_{x}+\lambda_{35} \dot{v}_{z}+\dot{\lambda}_{35} v_{z}-\left[\mu_{35} \eta_{b} \cos \varphi+\lambda_{26} v_{y}+\right.$

$\left.+\mu_{26} \eta_{b} \cos \varphi\right) \dot{\theta}-\left(\dot{\mu}_{35} \eta_{b} \cos \varphi+\mu_{35} \dot{\eta}_{b} \cos \varphi\right) \theta+\left[\mu_{24} \eta_{b} \cos \varphi-\left(m z_{c}-\lambda_{24}\right) v_{y}\right] \dot{\varphi}+$

$+\mu_{35} \dot{\varsigma}_{b}+\dot{\mu}_{35} \varsigma_{b}=M_{y}$,

$\left(J_{z}+\lambda_{66}\right) \ddot{\varphi}+\left(\dot{J}_{z}+\dot{\lambda}_{66}-\mu_{26} \eta_{b} \sin \varphi+\lambda_{26} v_{x}\right) \dot{\varphi}+\left(\dot{\mu}_{26} \eta_{b}+\mu_{26} \dot{\eta}_{b}-\mu_{24} \eta_{b} \dot{\psi}+\right.$

$\left.+\mu_{22} \eta_{b} v_{x}\right) \cos \varphi+\lambda_{26} \dot{v}_{y}+\left[\dot{\lambda}_{26}-\lambda_{24} \dot{\psi}+\left(\lambda_{22}-\lambda_{11}\right) v_{x}\right] v_{y}+\left(\mu_{26} \varsigma_{b}+\dot{\lambda}_{35} v_{z}+\right.$

$\left.+\mu_{35} \varsigma_{b}+\lambda_{24} v_{x}\right) \dot{\theta}+\left[\dot{\mu}_{26} \varsigma_{b}+\mu_{26} \dot{\varsigma}_{b}+\left(\mu_{22} \varsigma_{b}+\mu_{24}\right) v_{x}\right] \theta=M_{z}$.

The system of equations(20) should be supplemented by equations for forced components $\varsigma_{b}, \eta_{b}$ of vertical and horizontal transverse motion of the ship on a regular excitement which can be obtained in an obvious way from relations (18)

$$
\begin{aligned}
& \dot{\eta}_{b}=-\sigma_{k} \eta_{0}(r, \sigma, \chi) \sin \sigma_{k} t, \\
& \dot{\zeta}_{b}=-\psi v_{x}+\theta v_{y}+v_{z}-\dot{z}_{c} .
\end{aligned}
$$

Here $\chi$ - the angle between the direction of wave propagation and the longitudinal axis of the vessel.

\section{Conclusion}

The system of integrated equations of drilling vessel changes, obtained above, is not an analogue of a real vessel, as the latter, depending on the type of operations performed, may have one of the four dynamic analogues corresponding to the stages(initial, preliminary, drilling, ultimate) of drilling activity. The equation $f$ drilling vessel movement for each of the above-mentioned periods may be obtained from the equations (22) and (23) as special cases.

\section{References}

[1] Fossen, Thor I.,Guidance and Control of Ocean Marine Vehicles. John Wiley and Sons Ltd. New York, 1994.

[2] Lewis, E.V., Principles of Naval Architecture vol III: Motions in Waves and Controllability. Society of Naval Architecture and Marine Engineers, New York,1988.

[3] Jensen, Andreas G. Fluid dynamic derivatives: marine and wind engineering approaches. Journal of Wind Eng. and Industrial Aerodynamics 69 (1997) 777-793.

[4] Abkowitz, M.A. Lectures on ship hydrodynamics- steering and manoeuvrability. Technical Report Rep.No. Hy-5. Hydro og Aerodynamisk Laboratorium, Lyngby, Denmark, 1975.

[5] Nesin Danilo Y., Dushko Veronica R. Numerical model of the large carrying capacity crane ship with the fully revolving topside. Procedia EngineeringVolume 100, 2015, Pages 1082-1091.

[6] Kramar V. The frequency method of analysis of stability of the multidimensional automatic control system. Annals of DAAAM for 2009 \& Proceedings of the 20th International DAAAM Symposium, Volume 20, Published by DAAAM International, Vienna, Austria, 2009 p-0769-0770.

[7] T. Perez, A. J. Sørensen, and M. Blanke. Marine Vessel Models in Changing Operational Conditions - A Tutorial. In Proceedings of the 16th IFAC Symposium on System Identi_cation, Newcastle, Australia, 2006.

[8] Blagoveshensky S.N. Handbook of statics and dynamics of the ship. Shipbuilding Publishers, St. Petersburg, Russia,1976. 